\title{
Computational inference of gene regulatory networks: approaches, limitations and opportunities
}

\author{
Michael Banf*, Seung Y. Rhee* \\ Department of Plant Biology, \\ Carnegie Institution for Science, \\ 260 Panama Street, 93405, Stanford \\ srhee@carnegiescience.edu,michael.banf@gmx.net
}

\begin{abstract}
Gene regulatory networks lie at the core of cell function control. In E. coli and S. cerevisiae, the study of gene regulatory networks has led to the discovery of regulatory mechanisms responsible for the control of cell growth, differentiation and responses to environmental stimuli. In plants, computational rendering of gene regulatory networks is gaining momentum, thanks to the recent availability of high-quality genomes and transcriptomes and development of computational network inference approaches.

Here, we review current techniques, challenges and trends in gene regulatory network inference and highlight challenges and opportunities for plant science. We provide plant-specific application examples to guide researchers in selecting methodologies that suit their particular research questions.

Given the interdisciplinary nature of gene regulatory network inference, we tried to cater to both biologists and computer scientists to help them engage in a dialogue about concepts and caveats in network inference. Specifically, we discuss problems and opportunities in heterogeneous data integration for eukaryotic organisms and common caveats to be considered during network model evaluation.
\end{abstract}

Keywords: computational systems biology, gene regulatory network inference,

${ }^{*}$ Corresponding authors

Preprint submitted to Journal of ${ }^{A} T_{E} X$ Templates

September 7, 2016

(C) 2016. This manuscript version is made available under the Elsevier user license http://www.elsevier.com/open-access/userlicense/1.0/ 
machine learning, heterogeneous data integration, gene network evaluation

\section{Introduction}

Gene regulatory networks are central to all biological processes of an organism. In their most basic form, they describe the complex web of transcription factor proteins that bind regulatory sequences of target genes in order to affect their spatial and temporal expression [1].

Understanding gene expression regulation has an immediate impact in biology and medicine as many traits and diseases are associated with mutations in regulatory sequences or dysfunctional transcriptional regulators [1]. In agriculture, changes in plant transcriptional regulation shaped modern crops such as maize, rice and wheat and enabled yield increases [2]. Elucidation of transcriptional regulatory systems could help improve metabolite production rates and resilience against environmental stresses [3, 4,

Owing to their sessile nature, plants are subject to variations in their environment that drive adaptation. Yet, how gene-regulatory networks are rewired 15 to control the adapted traits or drive adaptation is largely unknown. Despite extensive insights into the core components of the transcriptional machinery, how specificity is encoded during the highly dynamic process of transcriptional regulation remains an open question [5].

Given the interdisciplinary nature of the problem, biologists and computer scientists need to engage in a dialogue to solve the network inference problem.

In this review, we aim to: i) introduce the basic concepts and procedures used for gene expression based regulatory network inference, which are borrowed from several disciplines including statistics, information theory, graph theory and machine learning; (ii) discuss limitations of network inference based only on transcriptional datasets; (iii) present data types and strategies used for integrative network inference and causal link predictions; and (iv) describe caveats and solutions for the evaluation and selection of statistically and biologically relevant regulatory interactions. 


\section{Methods for gene regulatory network inference from expression data}

Gene expression data generated by high-throughput technologies such as microarray or RNAseq still serve as one of the main sources for the development of gene regulatory networks. Therefore, we start our review by highlighting some of the main concepts, methods and limitations in inferring regulatory networks from different types of gene expression data. For an in-depth comparison of the state-of-the-art network inference methods using expression data, we refer the reader to [6, 7, 8, 9]. For an overview on gene expression normalization, filtering and pre-processing steps for network inference, see [10, 11].

\subsection{Correlation and information theoretic approaches}

Approaches within this category employ statistical analyses of dependencies between expression patterns. The most basic models, called co-expression networks, exploit correlations between expression profiles of genes [12]. A popular correlation measure is Pearson's correlation coefficient $r$ [10, i.e.

$$
r_{E_{i}, E_{j}}=\frac{\operatorname{cov}\left(E_{i}, E_{j}\right)}{\sigma\left(E_{i}\right) \cdot \sigma\left(E_{j}\right)}
$$

Here, $E_{i}, E_{j}$ denote gene expression profiles of two genes $i, j$ with covariance $\operatorname{cov}\left(E_{i}, E_{j}\right)$ and standard deviations $\sigma($.$) . Other correlation measures include$ Spearmans correlation [10] or the more recently introduced weighted correlation coefficient [13]. More sophisticated correlation based approaches aim to distinguish direct from indirect, spurious correlations between genes by using partial correlations [14, 15, 16].

Information theoretic concepts [17, 18] extend correlation to capture more complex statistical dependencies between expression patterns. This approach led to the development of a specific kind of association network called a relevance network [19]. Relevance networks define relationships between two genes $i, j$ based on an information theoretic property, called mutual information [19, 
based on their respective gene expression profiles $E_{i}$ and $E_{j}$. Mutual information is defined as:

$$
I\left(E_{i}, E_{j}\right)=\sum_{e_{i} \in E_{i}} \sum_{e_{j} \in E_{j}} p\left(e_{i}, e_{j}\right) \log \left(\frac{p\left(e_{i}, e_{j}\right)}{p\left(e_{i}\right) p\left(e_{j}\right)}\right)
$$

where $p\left(e_{i}, e_{j}\right)$ is the joint probability distribution of $E_{i}$ and $E_{j}$, and $p\left(e_{i}\right)$ and $p\left(e_{j}\right)$ denote the marginal probabilities.

Relevance networks are built by first constructing a fully connected graph for all gene pairs using mutual information to weight each link. Links whose associated weights lie below a certain threshold are removed from the network. The threshold, according to Butte and Kohane [19], is estimated by first randomizing the expression data, and then re-computing mutual information values to obtain a reference null distribution.

Various refinements of this idea have been proposed to discriminate direct from indirect effects [17, 20, 18, 12, 21. The most prominent methods include ARACNE (algorithm for the reconstruction of accurate cellular networks) [22], CLR (context likelihood of relatedness) [21], MRNET (minimum redundancy, maximum relevance) [20] and C3NET (conservative causal core) [17].

The ARACNE algorithm [22] adjusts the construction of a relevance network by applying a constraint known as Data Processing Inequality to filter indirect interactions. The Data Processing Inequality states that, if gene $i$ interacts with gene $j$ via gene $k$, then the following inequality holds with respect to their corresponding mutual information values: $I\left(E_{i}, E_{j}\right) \leq \min \left(I\left(E_{i}, E_{j}\right), I\left(E_{i}, E_{k}\right)\right)$,

75 i.e. the smallest of the mutual information scores $I(\cdot)$ within this inequality indicates an indirect regulatory interaction 22. As a consequence, ARACNE evaluates all possible gene triplets and prunes individual (indirect) interactions within each triplet, if the Data Processing Inequality is violated beyond a certain tolerance threshold.

80 The CLR algorithm [21] first estimates the pair-wise mutual information values for all gene pairs. Then, it estimates the statistical likelihood of each mutual information value $I_{i j}$ for a particular pair of genes $(i, j)$ by comparing this mutual information value to a background distribution. For each gene pair 
$(i, j)$, two z-scores are obtained, one for gene $i$ and one for gene $j$, by comparing Here lies CLR's major advantage over the relevance network approach by Butte and Kohan [19] or ARACNE, as individual thresholds can be established by considering an individual background for each pair of genes. This is in contrast to relevance networks or the ARANCE approach, which use a global threshold for graph pruning.

The MRNET algorithm [20] incorporates a feature selection methodology, called Minimum Redundancy Maximum Relevance (MRMR), to infer interactions between genes. This algorithm first places each gene as a target gene with all remaining genes as its putative regulators. The MRMR method is then 95 applied to select the best subset of regulators.

More recently, C3NET [17] has been proposed. This approach consists of two main steps. First, a relevance network is constructed and non-significant edges are pruned according to a chosen significance level. Then, only the most significant link for each gene, i.e. the highest mutual information value among the neighboring edges, is selected. This implies that the highest possible number of edges that can be inferred by C3NET is equal to the number of genes in the network.

In addition to aforementioned variations of the relevance network approach, concepts such as three-way and conditional mutual information have been proposed to directly address the problem of indirect interactions within gene triplets. For instance, the MI3 algorithm [23] uses three-way mutual information for inference, hypothesizing that gene regulation commonly involves more than one regulatory gene. Soranzo et al. [12] use only the conditional mutual information (CMI) to infer regulatory networks between gene triplets, pruning links that fall below a chosen threshold.

\subsubsection{Limitations}

In general, co-expression and relevance networks are designed to help explore co-functionality of genes on a systems level [13]. In this context, the no- 
tion of separating direct from indirect regulatory effects between genes should information 22, was used to construct an A. thaliana root transcriptional regulatory network [36. The predicted network successfully recovered previously 
identified root transcriptional modules as well as proposed new transcription factors involved in the regulation of root secondary cell-wall synthesis. The concept of partial correlations was used to analyze expression time series datasets in order to investigate the impact of the diurnal cycle on starch metabolism regulation in A. thaliana [35].

\subsection{Boolean network approaches}

Boolean networks represent relationships among regulators and putative target genes based on Boolean logic functions [37, 38. Boolean networks assume each gene $g$ at a time point $t$ to be in one of two states, active or inactive, according to its gene expression profile at time $t$. Hence, the gene expression profiles of all genes are discretized before network inference. The challenge of generating a Boolean network is to find a Boolean function per gene $g$ so that the observed, discrete data can be explained by the network model. One of the earliest algorithms for Boolean network inference is REVEAL (REVerse Engineering ALgorithm) [39].

\subsubsection{Limitations}

Since gene expression patterns are generally more complex than having only two states, data discretization often leads to information loss. In addition, being time-dependent, Boolean networks suffer from the small number of time points in gene expression time-series, making large-scale applications difficult. Nevertheless, Boolean networks can be interpreted intuitively. In addition, various extensions have been proposed to allow for multiple gene expression states and stochastic events during gene regulation 38, which are promising directions.

\subsubsection{Application examples in plant science}

Several examples of network inference using Boolean networks to describe small-scale $A$. thaliana developmental regulatory networks are provided in [40]. For instance, Savage et al. 41] used a Boolean framework to explore the regulatory mechanisms underlying patterning of $A$. thaliana root epidermis. They developed two competing network models describing different transcription factor 
interactions to regulate the patterning process. While one model proposed selfactivation feedback loop mechanism for one of the transcription factors involved, the other described to a mutual support mechanism between the transcription factors. These simulations were then used to design multiple experiments that led to the confirmation of the mutual support mechanism.

\subsection{Bayesian network approaches}

Bayesian networks were among the first approaches for generating gene regulatory networks from gene expression data [42, 43]. Bayesian networks represent conditional dependencies between random variables, genes $x_{i} \in X$ (nodes), using a directed acyclic graph structure $G(X, E)$. Edges $E$ between nodes indicate putative causal dependencies given their gene expression levels $[8]$.

$$
P\left(x_{1}, x_{2}, \ldots, x_{n}\right)=\prod_{i=1}^{n} P\left(x_{i} \mid P a\left(x_{i}\right)\right)
$$

The random variables are drawn from conditional probability distributions $P\left(x_{i} \mid P a\left(x_{i}\right)\right)$, where $\mathrm{Pa}\left(x_{i}\right)$ is a set of parents for each node $x_{i}$. A Bayesian network implicitly encodes the Markov assumption which states that, given its parents, each variable is independent of its non-descendants. Based on the Markov assumption, a Bayesian network specifies a decomposition of the joint distribution over all random variables down to the conditional distribution for each node. This probabilistic framework is appealing for modeling causal relationships [44].

Modeling using Bayesian networks involves two steps: (i) model selection, also referred to as structure learning; and (ii) parameter learning. Model selection involves constructing a specific network structure to identify the network topology that best explains the observed data from all possible network structures. In contrast, parameter learning involves estimating probability values associated with each node in the network. For model evaluation, Bayesian scoring metrics such as Bayesian information criterion (BIC) or Akaikes information criterion (AIC) are used 45. For each possible model, the metric defines a score that represents the logarithm of the probability that the model correctly describes a given set of data. 


\subsubsection{Limitations}

A fundamental practical problem of learning the structure of Bayesian networks is that the number of possible network topologies increases super-exponentially with the number of genes, which makes it not feasible to compute the likelihood

of all possible networks [46]. Hence, exact Bayesian network inference methods are applicable only to small networks or networks with built-in constraints. An example of such a heuristic would be to place an upper limit on the number of parents per node [47]. In this case, locally constrained search techniques can be applied, which make the computational complexity manageable 42. Other approaches include approximate sampling strategies such as Markov Chain Monte Carlo (MCMC) [48].

Traditional Bayesian models do not allow for modeling cycles, i.e. regulatory feedback loops, which occur frequently in biological networks [49. To circumvent this limitation, dynamic Bayesian networks were introduced [50, 51, whose main rationale is to unfold cyclic processes by mapping them onto a sequence of acyclic events 52. A prominent example of this category are variational Bayesian state space models (VBSSM) [53, 8, .

\subsubsection{Application examples in plant science}

Needham et al. applied a Bayesian network approach to learn the most plausible A. thaliana network structure based on a large compendium of individual gene expression datasets [54]. Their model was validated based on its ability to recover a well-studied regulatory network of the circadian clock. Penfold and colleagues [55] applied variational Bayesian state space models to infer gene regulatory networks across multiple experimental conditions in A. thaliana. They used their model to integrate complementary data from several stress conditions and to infer potential transcriptional switches in A. thaliana stress response. An in-depth comparison between different Bayesian network variations for inferring a network controlling lateral root initiation in A. thaliana was shown recently 8. 


\subsection{Regression and differential equation based models}

Regression-based approaches to gene regulatory network inference are based on the assumption that the expression profiles of the transcription factors that directly regulate a target gene are the most informative, among all transcription factors, to predict the expression profile of the target gene. In this respect, gene regulatory network inference is reformulated as a feature selection problem that seeks to find the most predictive subset of transcription factors for each target gene. In order to infer the global gene regulatory network, a regression model is formulated to predict a set of putative direct regulators among all transcription factors $r_{1}, \ldots r_{N}$ for each target gene $g$ :

$$
\sum_{i \in M}\left(e_{i}^{g}-f\left(\mathbf{e}_{i}^{r_{1}, \ldots, r_{N}}\right)\right)^{2},
$$
gene.

Recently, tree-based ensemble regression methods have been proposed 60. 61, 62, 63, including the GENIE3 algorithm 60. In contrast to linear regression models, tree-based approaches do not make any assumptions about the nature

Random forest regression is one of the most prominent examples of a tree based approach 64]. The main rationale is that for each gene $g$, a number of 
decision trees are grown over different bootstrapped samples of the complete

in time. A benefit of differential equation models is that they can be approximated as discrete-time (linear) models, also known as difference equations 68,

where $\alpha_{g}$ denotes the basal transcription rate, $f_{g}$ is an unknown regulation function and $\lambda_{g}$ is the decay rate of the transcript. Specific forms of the node functions $f_{g}($.$) typically come from biochemical considerations and modeling$ components of the genetic system 65. In general, these functions are non-linear because, among other things, all concentrations become saturated at some point 
which makes them solvable based on well-established methods of linear algebra, such as singular value decomposition (SVD) 68.

\subsubsection{Limitations}

Linear models yield reasonable predictions when the gene expression experiment is performed in a slowly changing system or around a steady state [69]. LASSO sparse regression tends to run the risk of producing inconsistent results with respect to feature (e.g. a regulator) selection. Therefore, a procedure known as stability selection, has been proposed within the TIGRESS algorithm [70.

\subsubsection{Application examples in plant science}

In-depth reviews about using dynamic approaches to analyze small-scale A. thaliana developmental regulatory networks are given in [71] and [4]. For instance, a linear regression model was used to refine an initial predicted regulatory network of the photosynthetic light acclimation response [72. In this approach, an initial network had been constructed based on Pearson's correlation. This network was subsequently pruned by applying a regression model on time-series gene expression datasets.

\section{Handling an under-determined problem: Caveats in gene regula- tory network inference based solely on gene expression data}

In this section, we discuss caveats of inferring gene regulatory networks from gene expression data alone. In the next section, we highlight one solution to the problem through integrating multiple, heterogeneous datasets.

\subsection{Gene expression and the curse of dimensionality problem}

Gene expression data can be divided into different experimental types such as steady state, time course or perturbation. Marbach et al. [7] compared different types of experimental data for their usefulness in regulatory network inference. They concluded that direct transcription factor perturbations such as 


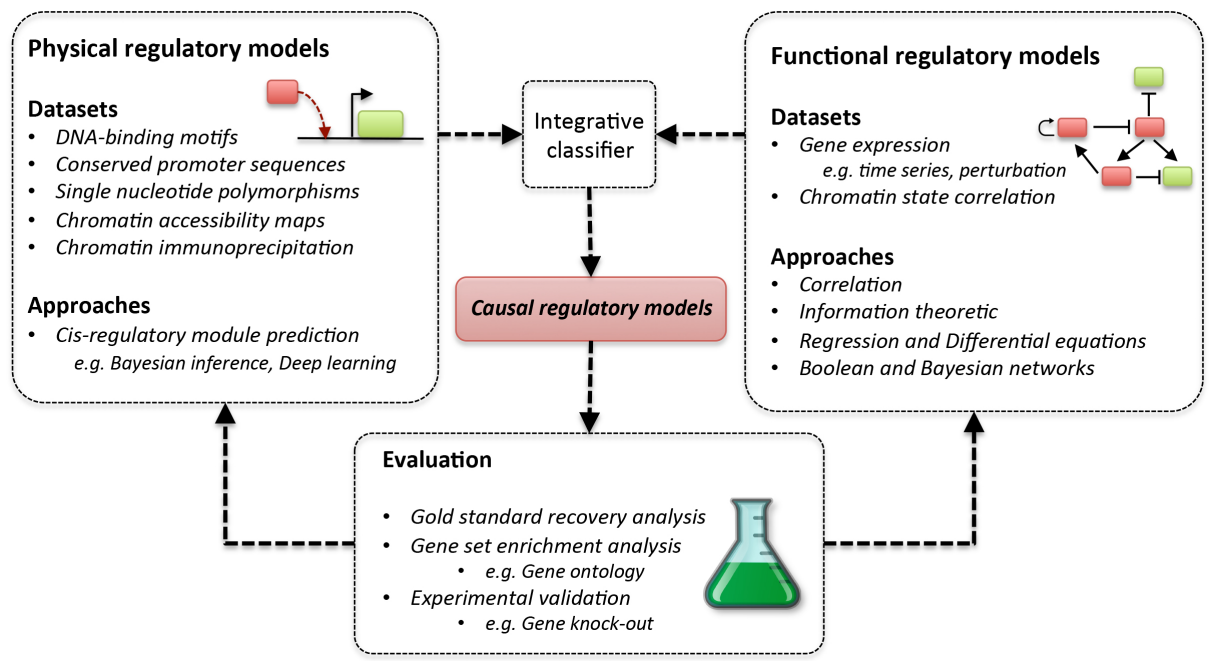

Figure 1: Integrating inferred (i) physical (DNA-binding) regulatory models and (ii) functional (influential) regulatory models to reconstruct condition-specific causal networks using an integrative classifier. Experimental validation can be used as a feedback mechanism to provide new datasets for network inference refinement and model training.

knockout or over-expression experiments, although typically sparse, are among the most informative data [7]. However, this particular type of experimental data often limits network inference to finding regulatory effects downstream of specific regulators [5, 73]. It does not allow the search for regulatory cascades upstream of certain target genes of interest [74. Furthermore, a given gene expression dataset drives the selection of a suitable method and scale of the inference. For instance, while Bayesian methods perform well on perturbation or time-course data for small regulatory sub-networks, they scored below average in a recent large-scale study on multi-factorial biological data in S. cerevisiae and E. coli [7].

In practice, all available types of gene expression data suffer from 'the curse of dimensionality', i.e. the number of genes (and in turn the number of putative regulatory interactions) dramatically outnumbers the number of gene expression samples [6, 32]. As a consequence, this may lead to many different Bayesian network structures or regression models that can explain the observed gene 

underlying regulatory network infeasible.

This high dimensionality problem is particularly acute for time-series data, which is appealing for establishing causal models of transcriptional regulation [67. This type of information is limited, especially in plant science, notwithrelated effects. To overcome this problem, time point interpolation approaches such as Gaussian process regression have been proposed for inferring a network to describe $A$. thaliana leaf senescence [77. In their study, Breeze et al. [77] used Gaussian process regression [78] as pre-processing step to interpolate

They then identified the rate of change in gene expression over time using the interpolated time points in order to cluster sets of co-regulated genes.

\subsection{The computational complexity of network structure learning}

Another challenge lies in the computational complexities of inference algorithms. For example, as already mentioned, network structure learning with Bayesian networks is limited, as the number of possible network topologies increases super-exponentially with the number of genes. This makes it not feasible to compute the likelihood of all possible networks [46]. As a consequence, problems such as Bayesian network structure learning, in complexity theory, are

lems. This means that these problems cannot be solved in polynomial time and can be used as a way of showing that certain classes of problems are not solvable in realistic time 79$]$. 


\subsection{Non-linearity and hidden layers of transcriptional regulation} 2.1) or linear regression approaches (Section 2.4) assume linear relationships between the expression levels of individual transcription factors and their putative targets. In contrast, information theoretic concepts (Section 2.1) or tree-based regression models (Section 2.4) do not make such an assumption, which makes tion, (partial) correlation as well as information theoretic approaches (Section 2.1) model individual relationships between a single regulator and its putative target. In contrast, Boolean (Section 2.2) and Bayesian networks (Section 2.3) as well as regression based approaches are capable of modeling combinatorial or epistatic effects of multiple transcription factors on a common target.

A bigger challenge is the general assumption that transcript levels of regulators and their targets display some degree of mutual dependency. It has recently been shown that this hypothesis cannot be taken for granted, especially for higher organisms. Studies by Kueffner et al. 80 and Marbach et factors and their known target genes tend to exceed the dependencies observed between non-interacting gene pairs for in silico and E. coli data but not for eukaryotic data such as those for S. cerevisiae [80]. In fact, the dependency distributions of non-interacting and interacting gene pairs for the $S$. cerevisiae data were found to be almost identical, underscoring the difficulty to detect true regulatory interactions based on dependencies derived from gene expression data alone. Similar observations were made for supervised plant developmental regulatory network inference in A. thaliana [81. The study by $\mathrm{Yu}$ et al. [81] proposed a supervised classification scheme to predict novel targets of known transcription factors by using gene expression profiles of known target genes as training set. In the study, the expression profiles of genes in positive and negative classes showed nearly identical distributions. This observation of almost identical distributions for non-interacting and interacting gene pairs can be explained, in part, by the roles of chromatin remodeling, post-transcriptional and 
to use other types of experimental data besides gene expression measurements [86, 87, 88. In the next section, we discuss different data types that have proven helpful for gene regulatory network inference, as well as strategies and caveats with respect to their integration for network inference.

\section{Gene regulatory network inference based on integration of multiple data types: Searching for causality}

In order to infer causal regulatory relationships between genes, Gardner and Faith [89] emphasize two complementary types of data-dependent inference 
strategies: (i) Physical models that describe real physical interactions, which are

(ii) Functional influence models that describe regulatory effects of a transcription factor on its putative downstream targets, including indirect influences. Reconstruction of high-quality, genome-scale regulatory relationships requires the integration of both physical and functional networks [87. Figure 1 illustrates such an integrative inference approach. Figure 1 also highlights the experimental feedback loop [74] to include experimental validation in the inference process. An early example of network inference to integrate physical and functional models is the combined use of gene expression data with gene promoter analysis to identify cis-regulatory elements of putative groups of potentially co${ }_{430}$ regulated genes [90, 31]. Examples in plant science include the reconstruction of a regulatory network for photosynthesis [91] and flower development [92. $\mathrm{Yu}$ et al. in 91 used a two-step pipeline to elucidate the detailed regulatory networks controlling the construction and maintenance of the photosynthetic machinery. First, the promoter regions of genes known for their involvement in photosynthesis were analyzed for known cis binding motifs to identify putative regulators. Subsequently, a mutual information based approach was used to determine putative interactions between these regulators and targets. In [92, Yu et al. derived a gene regulatory network of A. thaliana flower development. Here they extended their earlier proposed classification framework [81] vector machine classifier model to identify novel targets of known transcription factors.

Here we present different data types that have proven helpful for gene regulatory network inference, as well as strategies and caveats with respect to their integration for causal gene regulatory network inference.

\subsection{Data types for integrative gene regulatory network inference}

The most crucial types of information for gene regulatory network inference are transcription factor DNA binding site information generally derived from 
chromatin immunoprecipitation experiments and predictions based on motif sequence matching [87. A disadvantage of transcription factor binding data is that it can generate many false positives if no additional filtering (e.g. based on expression data) is applied [87. However, given that multiple transcription factors are often capable to bind the same motif, expression based filtering might also disregard true binding predictions. This would be the case if one of the transcription factor binding is not active within a specific expression experiments, and, as a consequence, lacks correlation with putative target's expression profile. In general, without any additional filtering, DNA binding information can be primarily harnessed to generate a regulatory blueprint i.e. a network of all possible regulatory interactions across different developmental stages or organ types (Figure 1, left). However, only a small subset of this space of possible regulatory relationships is active and observable in a specific condition (Figure 1. right). Accordingly, Mejia-Guerra et al. 74] coined the term gene regulatory grid in order to distinguish this static regulatory blueprint of all theoretically possible interactions from the highly dynamical nature of a condition-specific gene regulatory network instantiation.

Both experimental and predicted DNA binding information are becoming available for plants $93,94,95,96,97,98,99,100$. In the most recent release of the JASPAR database of experimentally validated binding motifs, the number of curated plant motifs was superseded only by that of vertebrates 95 . 470 The most recent release of the PlantPAN (Plant Promoter Analysis Navigator) database 99] reported binding motifs for 17673 transcription factors across 76 plant species. However, the majority of PlantPAN's DNA binding motif datasets are based on predictions that have not (yet) been described within plants and some predictions have even been inferred from information in differ475 ent kingdoms. In contrast, purely experimental motif datasets for A. thaliana are available for about 530 out of the approx. 2000 transcription factors [93, 94, 95, 96, 97, 98, 99, 100, 101]. Given that a typical binding element is $6-12 \mathrm{bp}$ long, the quest for functional transcription factor binding motifs in a 3000bp promoter region is prone to a high number of false positive predictions. This 


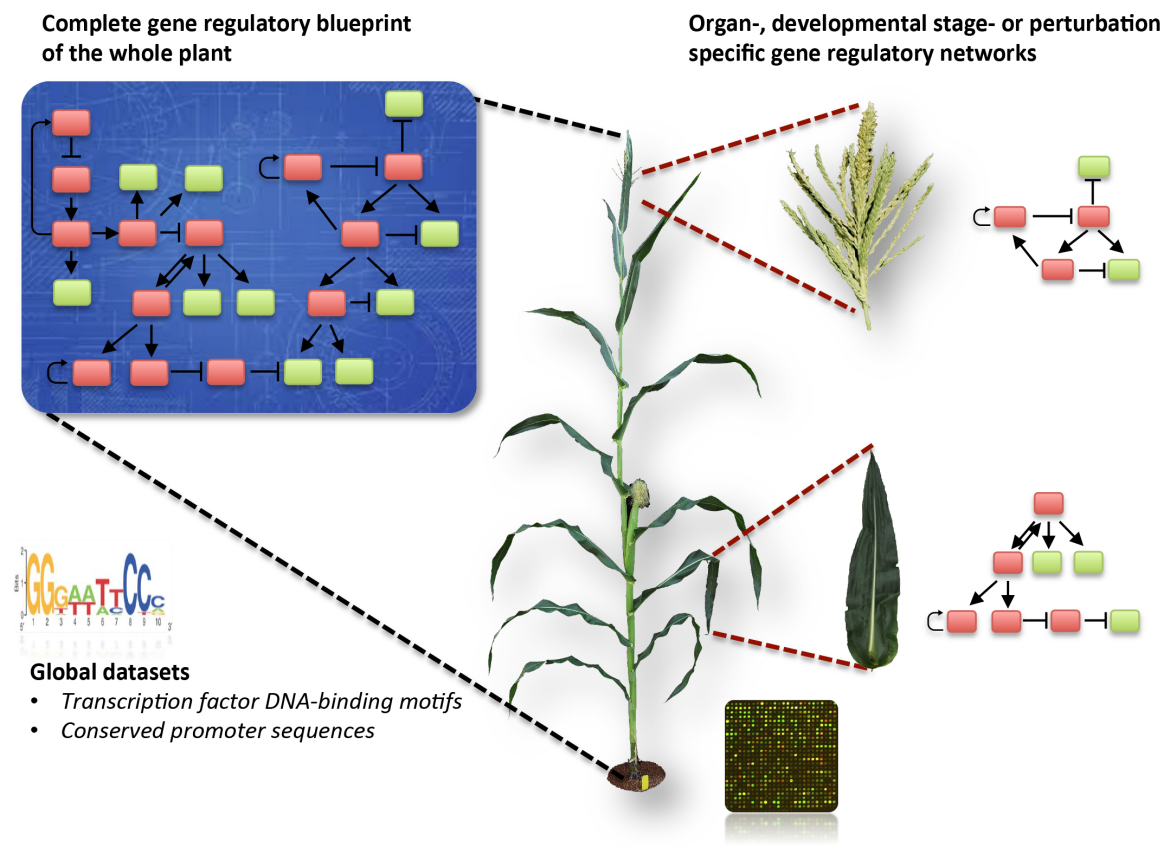

Figure 2: DNA binding information generates a regulatory blueprint, i.e. a network of all possible regulatory interactions between transcription factors (red) and other genes (green) across different developmental stages or organ types (left). Only a small subset of this space of possible regulatory relationships is active in a specific condition (right).

makes computational identification of cis-regulatory elements on a genome-scale challenging [102, 103, 104, 105].

Since transcriptional regulation of a target gene is often orchestrated by the combinatorial action of several transcription factors [104, the inference of cisregulatory modules, i.e. combinations of individual cis-regulatory elements, can lead to more robust predictions than single-element inference [106, 107]. This allows the incorporation of additional predictive features such as the order, relative spacing or distance to the transcription start site of the cis-elements [108]. An example for supervised cis-regulatory module prediction in A. thaliana is given in [109]. Recent advances in deep learning [107] have been applied to the identification of regulatory elements. Deep learning refers to neural networks with more than one hidden layer. The depth of these types of multi-layered 
neural networks allows for the construction of feature sets of increasing abstraction, which is particularly suited for learning complex representation and patterns such as combinatorial regulatory elements [107.

A large-scale comparison of cis-regulatory module detection approaches concluded that methods considering evolutionary conservation have a stronger predictive power than methods designed to be run on a single genome [110. In addition, conserved non-coding sequences in promoter regions are reliable pointers to regulatory elements controlling gene expression [87, 111]. Recent examples of conserved regulatory element recovery in plant science include comparative, phylogenetic footprinting approaches by [112, 113, 111. Yu et al. studied the positional distribution of transcription factor binding sites in A. thaliana and three additional closely related species [114]. The key assumption in these approaches is that mutations within functional regions of genes are likely to accumulate more slowly than those in regions without sequence-specific function and, therefore, the comparison of conserved sequences from orthologous genes can indicate segments that might direct transcription [102. These approaches predicted hundreds of conserved non-coding sequences upstream of $A$. thaliana genes. The length, specific positioning and enrichment for transcription factor binding sites suggest these conserved non-coding sequences play a functional role in transcriptional regulation. Other plant specific examples of conserved non-coding sequence inference include [115, 116].

Besides conserved non-coding sequences, the elucidation of expression Quantitative Trait Loci (eQTL) in the form of single nucleotide polymorphisms 515 (SNPs) and small insertions and deletions (INDELs) appears promising for identifying functional binding sites, given their enrichment for transcription factor binding sites [117, 93. A recent example in A. thaliana suggests a certain pathogen response to be dependent on a SNP present in a specific target genes promoter regulated by the transcriptional repressor VNI2 [93].

520 Another type of experimental data that can assist gene regulatory network inference is chromatin conformation mapping data from sequencing approaches such as DNase-seq, ATAC-seq and FAIREseq [118. These experimental meth- 
ods reveal regions of open chromatin, which is accessible to regulatory proteins [119, 120]. An overview of these methods is given in [118. For example, DNase I hypersensitivity site information has been used to validate predicted gene regulatory networks in A. thaliana [113. In their study, Van de Velde et al. observed that two-thirds of the regulators analyzed acted through binding events located in conserved non-coding regions coinciding with DNase I hypersensitive sites [113. In A thaliana, mapping of DNase I hypersensitivity sites from leaf and floral tissues has been useful for identifying cis-regulatory elements and elucidating the roles of transcription factors on leaf patterning and flower development [94. Recently, efforts to curate plant-specific open chromatin maps have begun [121. In addition, correlations between transcription factors and putative targets in chromatin modification [122] have been used to infer regulatory relationships between regulators and their target genes [87.

\subsection{Supervised versus unsupervised approaches for dataset integration}

The selection of an appropriate integrative classification framework to infer causal relationships remains challenging [88, 123, 124, 63]. Recent work on $D$. melanogaster demonstrated that multiple datasets can be integrated in a supervised fashion by learning the importance of each dataset in network inference 87. In general, a great variety of supervised machine learning methods exists, including Support Vector Machines, Random Forests and Neural Networks [125. In their analysis using D. melanogaster data, Marbach et al. showed that even a much simpler binary classifier (e.g. a logistic regression-based generalized linear model) can perform supervised data integration in inferring gene regulatory networks [87]. Interestingly, a simple, unsupervised feature aggregation yields similar results to supervised feature importance learning [87]. Alternatively, pipeline approaches, such as the INFERELATOR [126, are useful for combining individual algorithms used for different data types.

The competitive results of unsupervised approaches are encouraging, especially for plant science where gold standard data for model training is scarce. In addition, as unsupervised approaches, except for a few model parameters, do 
not require any training of a large set of weight parameters for data integration, they are less prone to overfitting. Overfitting occurs when a supervised model becomes too complex, i.e. it has too many parameters relative to the number of observations in the training data. Such a model has poor predictive performance on an unseen test set, as it is extensively tuned towards the training examples, and, as a consequence, overreacts to minor fluctuations from the training examples as given through unseen test examples.

\subsection{Caveats in dataset integration}

The consistency of the heterogeneous datasets is another important element in data integration. In contrast to working with prokaryotic organisms, datasets from multicellular organisms represent different developmental stages, organ/tissue/cell types and treatment conditions. Therefore, it is important to integrate only the same condition or organ/tissue/cell-specific data when constructing a specific regulatory network [87, or combine condition-independent data such as transcription factor binding predictions with more condition-specific data, as in [5, 127].

The integration of multiple inference methods on gene expression data has also been made [7. Examples in plant science include the inference of gene regulatory networks for oxidative stress [128] and secondary cell wall synthesis [129. However, a recent analysis on network-based gene function inference highlights that, while data aggregation exhibits enormous improvements, algorithm aggregation provides only modest benefits [130]. Therefore, algorithm aggregation 575 might provide an additional layer of robustness when gold standard data for evaluation and validation of individual methods is limited.

\section{Assessing the quality of predicted gene regulatory networks}

An important aspect of gene regulatory network inference is the experimental and in silico validation of the predicted regulatory relationships. Experimental validation can include a variety of approaches, including genetic and chemical 
perturbations of the regulators and observing changes of target gene expression and have been discussed in [131. Here we focus our discussion on in silico validation. A general approach for evaluating the quality of an inferred network in silico is to interpret the problem as a binary classification task. Therefore, edges are evaluated as being present or absent. Standard performance metrics such as receiver operating characteristic or precision versus recall curves are used to measure algorithms performance [132]. Precision $(P)$ and recall $(R)$ are defined as $P=\frac{T P}{T P+F P}$ and $R=\frac{T P}{T P+F N}$, respectively. $T P$ and $F P$ denote the number of true and false positives, while $F N$ is the number of false negatives. The receiver operator characteristic metric highlights how the number of correctly classified positive examples varies with the number of incorrectly classified negative examples. Although receiver operator curves are typically recommended when evaluating binary decision problems, they can paint an overly optimistic picture of an algorithm's performance if there is a large skew in the class distribution [133. Since this is the case for gene regulatory network inference where the number of putative true negatives by far outnumbers the most likely true positives, precision versus recall is generally more appropriate than receiver operator characteristic 88 .

Here, we focus on three major challenges to consider when treating gene regulatory network evaluation as a binary classification problem. First is the general lack of negative data in gold standard data that consists of manually curated, experimentally proven regulatory relationships. Therefore, these curated lists typically only contain positive class labels. Several strategies have been proposed to generate negative samples, including (i) random selection of regulator and target gene pairs; (ii) selecting genes without known binding sites of specific transcription factors in their promoters [134] as well as (iii) bootstrapping [135]. These samples, however, are not equivalent to true, biologically validated non-interaction and likely include false negatives.

Second, a major challenge lies in the extreme sparsity of gold standard data 610 for model training and evaluation. As a consequence, precision values rapidly approximate scores expected by random guessing with increasing recall and the 
highest precision value achieved by an algorithm is often defined by only few (e.g. 1-10) recalled gold standard links. This low density of recalled links over all predictions typically renders statistical significance tests between different inference methods meaningless. It also means that many computational models are likely to perform similarly with respect to the sparse gold standard.

Finally, curated gold standard data contain experimentally validated regulatory links with respect to a plethora of experimental conditions. Given a specific condition under scrutiny, it is unlikely that the entire gold standard will be recovered during inference. Accordingly, to exclude false positives, only a subset of the gold standard data, those corresponding to the conditions of the datasets employed for inference, can be used for model evaluation.

In lieu of substantial gold standard data, it is becoming common practice to assess the performance of an inference method with respect to the biological relevance of the inferred network [18, 87. An example of this approach is given for conserved regulatory network inference in A. thaliana [113. In general, it is assumed that genes in a gene regulatory network are preferentially linked to genes involved in similar biological processes [136. An example of how this assumption can be implemented to evaluate gene regulatory networks is given in 87. Here the number of resulting co-regulated gene pairs with similar experimental co-function annotation (Gene Ontology) is used to evaluate the biological relevance of a specific inference method. Co-regulation is defined as gene pairs being regulated by shared regulators (generally defined by a Jaccard's coefficient higher than $50 \%$ ) 87. Here, the Jaccard's coefficient computes the intersection (shared) over the union of involved regulators per gene pair. An evaluation strategy that utilizes biological information to assess the performance of an inference method could help quantify the biological relevance of the inferred network. Such a strategy will help distinguish multiple methods, which perform similarly on the regulatory evidence alone, and assist in the selection ${ }_{640}$ of more biologically relevant sub-networks for experimental validation. Validation could provide larger and more accurate gold standard data, which, in turn, could serve as training sets to improve prediction algorithms [5, 137]. 


\section{Conclusion}

With the emergence of large-scale transcriptional datasets, the mathematical

645

the gene regulatory code. Computational inference of gene regulatory networks can help prescreen in silico potential interactions and thus limit the extent of experimentation needed [27. With the advent of more powerful inference frameworks such as deep learning [107, the infrastructure needed for large-scale 


\section{Acknowledgements}

Funding: M.B. is a Feodor Lynen Postdoctoral Fellow supported by the Alexan-

der Humboldt Foundation. This work was supported in part by the Carnegie

Institution for Science and grants from the National Science Foundation (IOS-

1026003) and Department of Energy (DE-SC0008769). We thank Thomas

Hartwig for providing images of Zea mays ecotype B73.

\section{Competing interests}

The authors declare that they have no competing interests.

\section{References}

[1] T. I. Lee, R. A. Young, Transcriptional regulation and its misregulation in disease, Cell 152 (6) (2013) 1237-51. doi:10.1016/j.cell.2013.02.014.

[2] R. S. Meyer, M. D. Purugganan, Evolution of crop species: genetics of domestication and diversification, Nat Rev Genet 14 (12) (2013) 840-52. doi:10.1038/nrg3605.

[3] A. Iwase, K. Matsui, M. Ohme-Takagi, Manipulation of plant metabolic pathways by transcription factors, Plant Biotechnology 26 (1) (2009) 2938.

[4] D. Muhammad, S. Schmittling, C. Williams, T. A. Long, More than meets the eye: Emergent properties of transcription factors networks in arabidopsis, Biochim Biophys Actadoi:10.1016/j.bbagrm.2016.07.017.

[5] G. Krouk, J. Lingeman, A. M. Colon, G. Coruzzi, D. Shasha, Gene regulatory networks in plants: learning causality from time and perturbation, Genome Biol 14 (6) (2013) 123. doi:10.1186/gb-2013-14-6-123.

[6] D. Marbach, R. J. Prill, T. Schaffter, C. Mattiussi, D. Floreano, G. Stolovitzky, Revealing strengths and weaknesses of methods for gene 
network inference, Proc Natl Acad Sci U S A 107 (14) (2010) 6286-91. doi:10.1073/pnas.0913357107.

[7] D. Marbach, J. C. Costello, R. Küffner, N. M. Vega, R. J. Prill, D. M. Camacho, K. R. Allison, DREAM5 Consortium, M. Kellis, J. J. Collins, G. Stolovitzky, Wisdom of crowds for robust gene network inference, Nat Methods 9 (8) (2012) 796-804. doi:10.1038/nmeth.2016.

[8] D. Muraro, U. Vo, M. Wilson, M. Bennett, H. Byrne, I. De Smet, C. Hodgman, J. King, Inference of the genetic network regulating lateral root initiation in arabidopsis thaliana, IEEE/ACM Trans Comput Biol Bioinform 10 (1) (2013) 50-60. doi:10.1109/TCBB.2013.3.

[9] S. R. Maetschke, P. B. Madhamshettiwar, M. J. Davis, M. A. Ragan, Supervised, semi-supervised and unsupervised inference of gene regulatory networks, Brief Bioinform 15 (2) (2014) 195-211. doi:10.1093/ bib/bbt034.

[10] B. Usadel, T. Obayashi, M. Mutwil, F. M. Giorgi, G. W. Bassel, M. Tanimoto, A. Chow, D. Steinhauser, S. Persson, N. J. Provart, Co-expression tools for plant biology: opportunities for hypothesis generation and caveats, Plant Cell Environ 32 (12) (2009) 1633-51. doi:10.1111/j. $1365-3040.2009 .02040 \cdot x$

[11] Y. Li, S. A. Pearl, S. A. Jackson, Gene networks in plant biology: Approaches in reconstruction and analysis, Trends Plant Sci 20 (10) (2015) 664-75. doi:10.1016/j.tplants.2015.06.013.

[12] N. Soranzo, G. Bianconi, C. Altafini, Comparing association network algorithms for reverse engineering of large-scale gene regulatory networks: synthetic versus real data, Bioinformatics 23 (13) (2007) 1640-7. doi:10.1093/bioinformatics/btm163.

[13] B. Zhang, S. Horvath, A general framework for weighted gene co- 
expression network analysis, Stat Appl Genet Mol Biol 4 (2005) Article17. doi:10.2202/1544-6115.1128.

[14] J. Schäfer, K. Strimmer, An empirical bayes approach to inferring largescale gene association networks, Bioinformatics 21 (6) (2005) 754-64. doi: 10.1093/bioinformatics/bti062

[15] J. Friedman, T. Hastie, R. Tibshirani, Sparse inverse covariance estimaロ tion with the graphical lasso, Biostatistics 9 (3) (2008) 432-41. doi: 10.1093/biostatistics/kxm045

[16] Z. Liu, S. Lin, N. Deng, D. P. B. McGovern, S. Piantadosi, Sparse inverse covariance estimation with 10 penalty for network construction with omics data, J Comput Biol 23 (3) (2016) 192-202. doi:10.1089/cmb.2015. 0102

[17] G. Altay, F. Emmert-Streib, Revealing differences in gene network inference algorithms on the network level by ensemble methods, Bioinformatics 26 (14) (2010) 1738-1744.

740 [18] F. Emmert-Streib, G. V. Glazko, G. Altay, R. de Matos Simoes, Statistical inference and reverse engineering of gene regulatory networks from

[1) observational expression data, Front Genet 3 (2012) 8. doi:10.3389/ fgene.2012.00008

[19] A. J. Butte, I. S. Kohane, Mutual information relevance networks: functional genomic clustering using pairwise entropy measurements, Pac Symp Biocomput (2000) 418-29.

[20] P. Meyer, Information-theoretic inference of gene networks using backward elimination, in: Conference on Bioinformatics and Computational Biology, 2010 .

[21] J. J. Faith, B. Hayete, J. T. Thaden, I. Mogno, J. Wierzbowski, G. Cottarel, S. Kasif, J. J. Collins, T. S. Gardner, Large-scale mapping and validation of escherichia coli transcriptional regulation from a 
compendium of expression profiles, PLoS Biol 5 (1) (2007) e8. doi: 10.1371/journal.pbio.0050008

[27] G. W. Bassel, A. Gaudinier, S. M. Brady, L. Hennig, S. Y. Rhee,
I. De Smet, Systems analysis of plant functional, transcriptional, physical

[22] A. A. Margolin, I. Nemenman, K. Basso, C. Wiggins, G. Stolovitzky, R. Dalla Favera, A. Califano, Aracne: an algorithm for the reconstruction of gene regulatory networks in a mammalian cellular context, BMC Bioinformatics 7 Suppl 1 (2006) S7. doi:10.1186/1471-2105-7-S1-S7.

[23] W. Luo, K. D. Hankenson, P. J. Woolf, Learning transcriptional regulatory networks from high throughput gene expression data using continuous three-way mutual information, BMC Bioinformatics 9 (2008) 467. doi: 10.1186/1471-2105-9-467.

[24] C. Kaleta, A. Göhler, S. Schuster, K. Jahreis, R. Guthke, S. Nikolajewa, Integrative inference of gene-regulatory networks in escherichia coli using information theoretic concepts and sequence analysis, BMC Syst Biol 4 (2010) 116. doi:10.1186/1752-0509-4-116

[25] M. Lopes, G. Bontempi, Experimental assessment of static and dynamic algorithms for gene regulation inference from time series expression data, Front Genet 4 (2013) 303. doi:10.3389/fgene.2013.00303

[26] P. Zoppoli, S. Morganella, M. Ceccarelli, Timedelay-aracne: Reverse engineering of gene networks from time-course data by an information theoretic approach, BMC Bioinformatics 11 (2010) 154. doi:10.1186/ 1471-2105-11-154. interaction, and metabolic networks, Plant Cell 24 (10) (2012) 3859-75. doi:10.1105/tpc.112.100776.

[28] P. Langfelder, S. Horvath, Eigengene networks for studying the relationships between co-expression modules, BMC Syst Biol 1 (2007) 54. doi:10.1186/1752-0509-1-54. 
[29] T. Michoel, R. De Smet, A. Joshi, Y. Van de Peer, K. Marchal, Comparative analysis of module-based versus direct methods for reverseengineering transcriptional regulatory networks, BMC Syst Biol 3 (2009) 49. doi:10.1186/1752-0509-3-49.

[30] C. Huttenhower, K. T. Mutungu, N. Indik, W. Yang, M. Schroeder, J. J. Forman, O. G. Troyanskaya, H. A. Coller, Detailing regulatory networks through large scale data integration, Bioinformatics 25 (24) (2009) 326774. doi:10.1093/bioinformatics/btp588

[31] D. J. Reiss, N. S. Baliga, R. Bonneau, Integrated biclustering of heterogeneous genome-wide datasets for the inference of global regulatory networks, BMC Bioinformatics 7 (2006) 280. doi:10.1186/ 1471-2105-7-280.

[32] R. De Smet, K. Marchal, Advantages and limitations of current network inference methods, Nat Rev Microbiol 8 (10) (2010) 717-29. doi:10. $1038 /$ nrmicro2419

[33] A. Joshi, R. De Smet, K. Marchal, Y. Van de Peer, T. Michoel, Module networks revisited: computational assessment and prioritization of model predictions, Bioinformatics 25 (4) (2009) 490-6. doi:10.1093/ bioinformatics/btn658.

[34] R. J. Schaefer, J.-M. Michno, C. L. Myers, Unraveling gene function in agricultural species using gene co-expression networks, Biochim Biophys Actadoi:10.1016/j.bbagrm.2016.07.016.

[35] R. Opgen-Rhein, K. Strimmer, From correlation to causation networks: a simple approximate learning algorithm and its application to highdimensional plant gene expression data, BMC Syst Biol 1 (2007) 37. doi:10.1186/1752-0509-1-37.

[36] R. A. Chávez Montes, G. Coello, K. L. González-Aguilera, N. MarschMartínez, S. de Folter, E. R. Alvarez-Buylla, Aracne-based inference, 
using curated microarray data, of arabidopsis thaliana root transcrip-

[37] P. D'haeseleer, S. Liang, R. Somogyi, Genetic network inference: from coexpression clustering to reverse engineering, Bioinformatics 16 (8) (2000) 707-26.

[38] I. Shmulevich, E. R. Dougherty, S. Kim, W. Zhang, Probabilistic boolean networks: a rule-based uncertainty model for gene regulatory networks, Bioinformatics 18 (2) (2002) 261-74.

[39] S. Liang, S. Fuhrman, R. Somogyi, Reveal, a general reverse engineering algorithm for inference of genetic network architectures, Pac Symp Biocomput (1998) 18-29.

[40] A. M. Middleton, E. Farcot, M. R. Owen, T. Vernoux, Modeling regulatory networks to understand plant development: small is beautiful, Plant Cell 24 (10) (2012) 3876-91. doi:10.1105/tpc.112.101840.

[41] N. S. Savage, T. Walker, Y. Wieckowski, J. Schiefelbein, L. Dolan, N. A. M. Monk, A mutual support mechanism through intercellular movement of caprice and glabra3 can pattern the arabidopsis root epidermis, PLoS Biol 6 (9) (2008) e235. doi:10.1371/journal.pbio.0060235.

[42] N. Friedman, M. Linial, I. Nachman, D. Pe'er, Using bayesian networks to analyze expression data, J Comput Biol 7 (3-4) (2000) 601-20. doi: $10.1089 / 106652700750050961$.

[43] F. Markowetz, R. Spang, Inferring cellular networks-a review, BMC Bioinformatics 8 Suppl 6 (2007) S5. doi:10.1186/1471-2105-8-S6-S5

[44] S. Aluru, Handbook of computational molecular biology, Chapman and Hall/CRC, Boca Raton, FL, 2006. 
[45] Z. Liu, B. Malone, C. Yuan, Empirical evaluation of scoring functions for bayesian network model selection, BMC Bioinformatics 13 Suppl 15 (2012) S14. doi:10.1186/1471-2105-13-S15-S14.

[46] D. Heckerman, D. Geiger, D. M. Chickering, Learning bayesian networks: The combination of knowledge and statistical data, Mach. Learn. 20 (3) (1995) 197-243. doi:10.1023/A:1022623210503.

[47] I. Tsamardinos, L. E. Brown, C. F. Aliferis, The max-min hill-climbing bayesian network structure learning algorithm, Mach. Learn. 65 (1) (2006) 31-78. doi:10.1007/s10994-006-6889-7.

[48] S. Tasaki, B. Sauerwine, B. Hoff, H. Toyoshiba, C. Gaiteri, E. Chaibub Neto, Bayesian network reconstruction using systems genetics data: comparison of mcmc methods, Genetics 199 (4) (2015) 973-89. doi:10.1534/genetics.114.172619.

[49] S. Mangan, U. Alon, Structure and function of the feed-forward loop network motif, Proc Natl Acad Sci U S A 100 (21) (2003) 11980-5. doi:10.1073/pnas.2133841100.

[50] D. Husmeier, Sensitivity and specificity of inferring genetic regulatory interactions from microarray experiments with dynamic bayesian networks, Bioinformatics 19 (17) (2003) 2271-82.

[51] M. Grzegorczyk, D. Husmeier, Improvements in the reconstruction of time-varying gene regulatory networks: dynamic programming and regularization by information sharing among genes, Bioinformatics 27 (5) (2011) 693-9. doi:10.1093/bioinformatics/btq711.

[52] D. Koller, N. Friedman, Probabilistic graphical models: principles and techniques, Adaptive computation and machine learning, MIT Press, Cambridge, MA, 2009.

[53] M. J. Beal, F. Falciani, Z. Ghahramani, C. Rangel, D. L. Wild, A bayesian approach to reconstructing genetic regulatory networks with 
hidden factors, Bioinformatics 21 (3) (2005) 349-56. doi:10.1093/ bioinformatics/bti014.

[54] C. J. Needham, I. W. Manfield, A. J. Bulpitt, P. M. Gilmartin, D. R. Westhead, From gene expression to gene regulatory networks in arabidopsis thaliana, BMC Syst Biol 3 (2009) 85. doi:10.1186/1752-0509-3-85

[55] C. A. Penfold, V. Buchanan-Wollaston, K. J. Denby, D. L. Wild, Nonparametric bayesian inference for perturbed and orthologous gene regulatory networks, Bioinformatics 28 (12) (2012) i233-41. doi:10.1093/ bioinformatics/bts222.

[56] M. A. Efroymson, Mathematical Methods for Digital Computers, John Wiley, New York, 1960, Ch. Multiple regression analysis, pp. 192-203.

[57] B. Efron, Least angle regression, Annals of Statistics 24 (2004) 507-451.

[58] A. E. Hoerl, R. W. Kennard, Ridge regression: biased estimation for nonorthogonal problems, Technometrics 12 (1970) 55-67.

[59] R. Tibshirani, Regression shrinkage and selection via the lasso, Journal of the Royal Statistical Society 58 (1996) 267-288.

[60] V. A. Huynh-Thu, A. Irrthum, L. Wehenkel, P. Geurts, Inferring regulatory networks from expression data using tree-based methods, PLoS One 5 (9). doi:10.1371/journal.pone.0012776.

[61] D. A. K. Maduranga, J. Zheng, P. A. Mundra, J. C. Rajapakse, Pattern Recognition in Bioinformatics: 8th IAPR International Conference, PRIB 2013, Nice, France, June 17-20, 2013. Proceedings, Springer Berlin Heidelberg, Berlin, Heidelberg, 2013, Ch. Inferring Gene Regulatory Networks from Time-Series Expressions Using Random Forests Ensemble, pp. 13-22. doi:10.1007/978-3-642-39159-0_2.

[62] V. A. Huynh-Thu, G. Sanguinetti, Combining tree-based and dynamical systems for the inference of gene regulatory networks, Bioinformatics 31 (10) (2015) 1614-22. doi:10.1093/bioinformatics/btu863. 
[63] F. Petralia, P. Wang, J. Yang, Z. Tu, Integrative random forest for gene regulatory network inference, Bioinformatics 31 (12) (2015) i197-205. doi:10.1093/bioinformatics/btv268.

q [64] L. Breiman, Random forests, Mach. Learn. 45 (1) (2001) 5-32. doi: 10.1023/A: 1010933404324

[65] N. Lawrence, G. Sanguinetti, M. Rattray, Modelling transcriptional regulation using gaussian processes, in: Conference on Neural Information Processing Systems, 2006.

[66] P. Gao, A. Honkela, M. Rattray, N. D. Lawrence, Gaussian process modelling of latent chemical species: applications to inferring transcription

1. factor activities, Bioinformatics 24 (16) (2008) i70-5. doi:10.1093/ bioinformatics/btn278.

[67] T. Aijo, H. Lahdesmaki, Learning gene regulatory networks from gene expression measurements using non-parametric molecular kinetics, Bioinformatics 25 (22) (2009) 2937-2944. arXiv:http://bioinformatics. oxfordjournals.org/content/25/22/2937.full.pdf+html, doi:10. 1093/bioinformatics/btp511.

[68] M. Bansal, G. Della Gatta, D. di Bernardo, Inference of gene regulatory networks and compound mode of action from time course gene expression profiles, Bioinformatics 22 (7) (2006) 815-22. doi:10.1093/ bioinformatics/btl003.

[69] V. Filkov, Handbook of Computational Molecular Biology, Chapman \& Hall/CRC Computer and Information Science Series, 2005, Ch. Identifying Gene Regulatory Networks from Gene Expression Data, pp. 27/1$27 / 30$.

[70] A.-C. Haury, F. Mordelet, P. Vera-Licona, J.-P. Vert, Tigress: Trustful inference of gene regulation using stability selection, BMC Syst Biol 6 (2012) 145. doi:10.1186/1752-0509-6-145 
[71] E. R. Alvarez-Buylla, M. Benitez, E. B. Davila, A. Chaos, C. EspinosaSoto, P. Padilla-Longoria, Gene regulatory network models for plant development, Curr. Opin. Plant Biol. 10 (1) (2007) 83-91. doi:10.1016/j . pbi.2006.11.008.

[72] C.-W. Yao, B.-D. Hsu, B.-S. Chen, Constructing gene regulatory networks for long term photosynthetic light acclimation in arabidopsis thaliana, BMC Bioinformatics 12 (2011) 335. doi:10.1186/1471-2105-12-335.

[73] A. Gaudinier, S. M. Brady, Mapping transcriptional networks in plants: Data-driven discovery of novel biological mechanisms, in: Annual Review of Plant Biology, Vol. 67, 2016.

[74] M. K. Mejia-Guerra, M. Pomeranz, K. Morohashi, E. Grotewold, From plant gene regulatory grids to network dynamics, Biochim Biophys Acta 1819 (5) (2012) 454-65. doi:10.1016/j.bbagrm.2012.02.016.

[75] J. Kilian, D. Whitehead, J. Horak, D. Wanke, S. Weinl, O. Batistic, C. D'Angelo, E. Bornberg-Bauer, J. Kudla, K. Harter, The atgenexpress global stress expression data set: protocols, evaluation and model data analysis of uv-b light, drought and cold stress responses, Plant J 50 (2) (2007) 347-63. doi:10.1111/j.1365-313X.2007.03052.x.

[76] O. Windram, P. Madhou, S. McHattie, C. Hill, R. Hickman, E. Cooke, D. J. Jenkins, C. A. Penfold, L. Baxter, E. Breeze, S. J. Kiddle, J. Rhodes, S. Atwell, D. J. Kliebenstein, Y.-S. Kim, O. Stegle, K. Borgwardt, C. Zhang, A. Tabrett, R. Legaie, J. Moore, B. Finkenstadt, D. L. Wild, A. Mead, D. Rand, J. Beynon, S. Ott, V. Buchanan-Wollaston, K. J. Denby, Arabidopsis defense against botrytis cinerea: chronology and regulation deciphered by high-resolution temporal transcriptomic analysis, Plant Cell 24 (9) (2012) 3530-57. doi:10.1105/tpc.112.102046.

[77] E. Breeze, E. Harrison, S. McHattie, L. Hughes, R. Hickman, C. Hill, S. Kiddle, Y.-S. Kim, C. A. Penfold, D. Jenkins, C. Zhang, K. Morris, 
C. Jenner, S. Jackson, B. Thomas, A. Tabrett, R. Legaie, J. D. Moore, D. L. Wild, S. Ott, D. Rand, J. Beynon, K. Denby, A. Mead, V. BuchananWollaston, High-resolution temporal profiling of transcripts during arabidopsis leaf senescence reveals a distinct chronology of processes and regulation, Plant Cell 23 (3) (2011) 873-94. doi:10.1105/tpc.111.083345

[78] C. E. Rasmussen, Gaussian Processes in Machine Learning, Springer Berlin Heidelberg, Berlin, Heidelberg, 2004, pp. 63-71. doi:10.1007/ 978-3-540-28650-9_4. URL http://dx.doi .org/10.1007/978-3-540-28650-9_4

[79] D. P. Bovet, P. Crescenzi, Introduction to the Theory of Complexity, Prentice Hall International (UK) Ltd., Hertfordshire, UK, UK, 1994.

[80] R. Küffner, T. Petri, P. Tavakkolkhah, L. Windhager, R. Zimmer, Inferring gene regulatory networks by anova, Bioinformatics 28 (10) (2012) 1376-82. doi:10.1093/bioinformatics/bts143.

[81] X. Yu, T. Liu, X. Zheng, Z. Yang, J. Wang, Prediction of regulatory interactions in arabidopsis using gene-expression data and support vector machines, Plant Physiol Biochem 49 (3) (2011) 280-3. doi:10.1016/j. plaphy.2011.01.002.

[82] K. L. MacQuarrie, A. P. Fong, R. H. Morse, S. J. Tapscott, Genomewide transcription factor binding: beyond direct target regulation, Trends Genet 27 (4) (2011) 141-8. doi:10.1016/j.tig.2011.01.001.

[83] B. Zhu, W. Zhang, T. Zhang, B. Liu, J. Jiang, Genome-wide prediction and validation of intergenic enhancers in arabidopsis using open chro970 ㅁ matin signatures, Plant Cell 27 (9) (2015) 2415-26. doi:10.1105/tpc. 15.00537 .

[84] A. P. Marand, T. Zhang, B. Zhu, J. Jiang, Towards genome-wide prediction and characterization of enhancers in plants, Biochim Biophys Actadoi:10.1016/j.bbagrm.2016.06.006. 
[89] T. S. Gardner, J. J. Faith, Reverse-engineering transcription control net-

[90] E. Segal, R. Yelensky, D. Koller, Genome-wide discovery of transcriptional modules from dna sequence and gene expression, Bioinformatics 19 Suppl 1 (2003) i273-82.

[91] X. Yu, G. Zheng, L. Shan, G. Meng, M. Vingron, Q. Liu, X.-G. Zhu,

[92] X. Yu, H. Gao, X. Zheng, C. Li, J. Wang, A computational method of predicting regulatory interactions in arabidopsis based on gene expression 1000

[85] C. D. Hirsch, N. M. Springer, Transposable element influences on gene expression in plants, Biochim Biophys Actadoi:10.1016/j.bbagrm.2016. 05.010

[86] H. Bolouri, Modeling genomic regulatory networks with big data, Trends Genet 30 (5) (2014) 182-91. doi:10.1016/j.tig.2014.02.005

[87] D. Marbach, S. Roy, F. Ay, P. E. Meyer, R. Candeias, T. Kahveci, C. A. Bristow, M. Kellis, Predictive regulatory models in drosophila melanogaster by integrative inference of transcriptional networks, Genome Res 22 (7) (2012) 1334-49. doi:10.1101/gr.127191.111

[88] M. Hecker, S. Lambeck, S. Toepfer, E. van Someren, R. Guthke, Gene regulatory network inference: data integration in dynamic models-a review, Biosystems 96 (1) (2009) 86-103. doi:10.1016/j.biosystems.2008.12. 004 works, Phys Life Rev 2 (1) (2005) 65-88. doi:10.1016/j.plrev.2005. 01.001 . Reconstruction of gene regulatory network related to photosynthesis in arabidopsis thaliana, Front Plant Sci 5 (2014) 273. doi:10.3389/fpls. 2014.00273

data and sequence information, Comput Biol Chem 51 (2014) 36-41. doi: 10.1016/j.compbiolchem.2014.04.003 
[93] M. T. Weirauch, A. Yang, M. Albu, A. G. Cote, A. Montenegro-Montero, P. Drewe, H. S. Najafabadi, S. A. Lambert, I. Mann, K. Cook, H. Zheng, A. Goity, H. van Bakel, J.-C. Lozano, M. Galli, M. G. Lewsey, E. Huang, T. Mukherjee, X. Chen, J. S. Reece-Hoyes, S. Govindarajan, G. Shaulsky, A. J. M. Walhout, F.-Y. Bouget, G. Ratsch, L. F. Larrondo, J. R. Ecker, T. R. Hughes, Determination and inference of eukaryotic transcription factor sequence specificity, Cell 158 (6) (2014) 1431-43. doi:10.1016/j. cell.2014.08.009

[94] J. M. Franco-Zorrilla, I. López-Vidriero, J. L. Carrasco, M. Godoy, P. Vera, R. Solano, Dna-binding specificities of plant transcription factors and their potential to define target genes, Proc Natl Acad Sci U S A 111 (6) (2014) 2367-72. doi:10.1073/pnas.1316278111.

[95] A. Mathelier, O. Fornes, D. J. Arenillas, C.-Y. Chen, G. Denay, J. Lee, W. Shi, C. Shyr, G. Tan, R. Worsley-Hunt, A. W. Zhang, F. Parcy, B. Lenhard, A. Sandelin, W. W. Wasserman, Jaspar 2016: a major expansion and update of the open-access database of transcription factor binding profiles, Nucleic Acids Res 44 (D1) (2016) D110-5. doi: 10.1093/nar/gkv1176.

[96] R. Hehl, L. Norval, A. Romanov, L. Bülow, Boosting athamap database content with data from protein binding microarrays, Plant Cell Physiol 57 (1) (2016) e4. doi:10.1093/pcp/pcv156.

[97] A. Yilmaz, M. K. Mejia-Guerra, K. Kurz, X. Liang, L. Welch, E. Grotewold, Agris: the arabidopsis gene regulatory information server, an update, Nucleic Acids Res 39 (Database issue) (2011) D1118-22. doi: 10.1093/nar/gkq1120.

[98] V. Matys, E. Fricke, R. Geffers, E. Gössling, M. Haubrock, R. Hehl, K. Hornischer, D. Karas, A. E. Kel, O. V. Kel-Margoulis, D.-U. Kloos, S. Land, B. Lewicki-Potapov, H. Michael, R. Münch, I. Reuter, S. Rotert, H. Saxel, M. Scheer, S. Thiele, E. Wingender, Transfac: transcriptional 
regulation, from patterns to profiles, Nucleic Acids Res 31 (1) (2003) 3748.

[99] C.-N. Chow, H.-Q. Zheng, N.-Y. Wu, C.-H. Chien, H.-D. Huang, T.-Y. Lee, Y.-F. Chiang-Hsieh, P.-F. Hou, T.-Y. Yang, W.-C. Chang, Plantpan 2.0: an update of plant promoter analysis navigator for reconstructing transcriptional regulatory networks in plants, Nucleic Acids Res 44 (D1) (2016) D1154-60. doi:10.1093/nar/gkv1035.

[100] R. C. O'Malley, S.-S. C. Huang, L. Song, M. G. Lewsey, A. Bartlett, J. R. Nery, M. Galli, A. Gallavotti, J. R. Ecker, Cistrome and epicistrome features shape the regulatory dna landscape, Cell 165 (5) (2016) 1280-92. doi:10.1016/j.cell.2016.04.038

[101] J. L. Pruneda-Paz, G. Breton, D. H. Nagel, S. E. Kang, K. Bonaldi, C. J. Doherty, S. Ravelo, M. Galli, J. R. Ecker, S. A. Kay, A genome-scale resource for the functional characterization of arabidopsis transcription

ㅁ factors, Cell Rep 8 (2) (2014) 622-32. doi:10.1016/j.celrep.2014.06. 033

[102] W. W. Wasserman, A. Sandelin, Applied bioinformatics for the identification of regulatory elements, Nat Rev Genet 5 (4) (2004) 276-87. doi:10.1038/nrg1315.

[103] M. Tompa, N. Li, T. L. Bailey, G. M. Church, B. De Moor, E. Eskin, A. V. Favorov, M. C. Frith, Y. Fu, W. J. Kent, V. J. Makeev, A. A. Mironov, W. S. Noble, G. Pavesi, G. Pesole, M. Régnier, N. Simonis, S. Sinha, G. Thijs, J. van Helden, M. Vandenbogaert, Z. Weng, C. Workman, C. Ye, Z. Zhu, Assessing computational tools for the discovery of transcription factor binding sites, Nat Biotechnol 23 (1) (2005) 137-44. doi:10.1038/ nbt1053.

[104] R. C. Hardison, J. Taylor, Genomic approaches towards finding cisregulatory modules in animals, Nat Rev Genet 13 (7) (2012) 469-83. doi:10.1038/nrg3242. 

cis-regulatory code, Trends Genet 29 (1) (2013) 11-22. doi:10.1016/j. tig.2012.09.007.

[106] L. Narlikar, I. Ovcharenko, Identifying regulatory elements in eukaryotic

[105] J. O. Yáñez-Cuna, E. Z. Kvon, A. Stark, Deciphering the transcriptional genomes, Brief Funct Genomic Proteomic 8 (4) (2009) 215-30. doi:10. 1093/bfgp/elp014.

[107] B. Alipanahi, A. Delong, M. T. Weirauch, B. J. Frey, Predicting the sequence specificities of dna- and rna-binding proteins by deep learning, Nat Biotechnol 33 (8) (2015) 831-8. doi:10.1038/nbt.3300

[108] M. Levo, E. Segal, In pursuit of design principles of regulatory sequences, Nat Rev Genet 15 (7) (2014) 453-68. doi:10.1038/nrg3684.

[109] S. J. Schultheiss, W. Busch, J. U. Lohmann, O. Kohlbacher, G. Rätsch, Kirmes: kernel-based identification of regulatory modules in euchroप matic sequences, Bioinformatics 25 (16) (2009) 2126-33. doi:10.1093/ bioinformatics/btp278.

[110] J. Su, S. A. Teichmann, T. A. Down, Assessing computational methods of cis-regulatory module prediction, PLoS Comput Biol 6 (12) (2010) e1001020. doi:10.1371/journal.pcbi.1001020.

[111] D. De Witte, J. Van de Velde, D. Decap, M. Van Bel, P. Audenaert, P. Demeester, B. Dhoedt, K. Vandepoele, J. Fostier, Blsspeller: exhaustive comparative discovery of conserved cis-regulatory elements, Bioinformatics 31 (23) (2015) 3758-66. doi:10.1093/bioinformatics/btv466.

[112] L. Baxter, A. Jironkin, R. Hickman, J. Moore, C. Barrington, P. Krusche, N. P. Dyer, V. Buchanan-Wollaston, A. Tiskin, J. Beynon, K. Denby, S. Ott, Conserved noncoding sequences highlight shared components of regulatory networks in dicotyledonous plants, Plant Cell 24 (10) (2012) 3949-65. doi:10.1105/tpc.112.103010. 
[113] J. Van de Velde, K. S. Heyndrickx, K. Vandepoele, Inference of transcriptional networks in arabidopsis through conserved noncoding sequence analysis, Plant Cell 26 (7) (2014) 2729-45. doi:10.1105/tpc.114. 127001.

[114] C.-P. Yu, J.-J. Lin, W.-H. Li, Positional distribution of transcription factor binding sites in arabidopsis thaliana, Sci Rep 6 (2016) 25164. doi:10. 1038/srep25164.

[115] G. Turco, J. C. Schnable, B. Pedersen, M. Freeling, Automated conserved non-coding sequence (cns) discovery reveals differences in gene content

1 and promoter evolution among grasses, Front Plant Sci 4 (2013) 170. doi: $10.3389 /$ fpls.2013.00170

[116] D. Burgess, M. Freeling, The most deeply conserved noncoding sequences in plants serve similar functions to those in vertebrates despite large differences in evolutionary rates, Plant Cell 26 (3) (2014) 946-61. doi: $10.1105 /$ tpc.113.121905

[117] J. J. B. Keurentjes, J. Fu, I. R. Terpstra, J. M. Garcia, G. van den Ackerveken, L. B. Snoek, A. J. M. Peeters, D. Vreugdenhil, M. Koornneef, R. C. Jansen, Regulatory network construction in arabidopsis by using genome-wide gene expression quantitative trait loci, Proc Natl Acad Sci U S A 104 (5) (2007) 1708-13. doi:10.1073/pnas.0610429104.

[118] C. A. Meyer, X. S. Liu, Identifying and mitigating bias in next-generation sequencing methods for chromatin biology, Nat Rev Genet 15 (11) (2014) 709-21. doi:10.1038/nrg3788.

[119] W. Zhang, T. Zhang, Y. Wu, J. Jiang, Genome-wide identification of regulatory dna elements and protein-binding footprints using signatures 【 of open chromatin in arabidopsis, Plant Cell 24 (7) (2012) 2719-31. doi: $10.1105 /$ tpc.112.098061 
[120] M. Tsompana, M. J. Buck, Chromatin accessibility: a window into the genome, Epigenetics Chromatin 7 (1) (2014) 33. doi:10.1186/ 1756-8935-7-33.

[121] T. Zhang, A. P. Marand, J. Jiang, Plantdhs: a database for dnase i hypersensitive sites in plants, Nucleic Acids Res 44 (D1) (2016) D1148-53. doi:10.1093/nar/gkv962

[125] Y. Saeys, I. Inza, P. Larrañaga, A review of feature selection techniques in bioinformatics, Bioinformatics 23 (19) (2007) 2507-17. doi:10.1093/ bioinformatics/btm344.

[126] R. Bonneau, D. J. Reiss, P. Shannon, M. Facciotti, L. Hood, N. S. Baliga, 1135 V. Thorsson, The inferelator: an algorithm for learning parsimonious regulatory networks from systems-biology data sets de novo, Genome Biol 7 (5) (2006) R36. doi:10.1186/gb-2006-7-5-r36.

[127] R. Pique-Regi, J. F. Degner, A. A. Pai, D. J. Gaffney, Y. Gilad, J. K. Pritchard, Accurate inference of transcription factor binding from dna 1140 sequence and chromatin accessibility data, Genome Res 21 (3) (2011) 447-55. doi:10.1101/gr.112623.110. 
[128] V. Vermeirssen, I. De Clercq, T. Van Parys, F. Van Breusegem, Y. Van de Peer, Arabidopsis ensemble reverse-engineered gene regulatory network discloses interconnected transcription factors in oxidative stress, Plant Cell 26 (12) (2014) 4656-79. doi:10.1105/tpc.114.131417

[129] M. Taylor-Teeples, L. Lin, M. de Lucas, G. Turco, T. W. Toal, A. Gaudinier, N. F. Young, G. M. Trabucco, M. T. Veling, R. Lamothe, P. P. Handakumbura, G. Xiong, C. Wang, J. Corwin, A. Tsoukalas, L. Zhang, D. Ware, M. Pauly, D. J. Kliebenstein, K. Dehesh, I. Tagkopoulos, G. Breton, J. L. Pruneda-Paz, S. E. Ahnert, S. A. Kay, S. P. Hazen, S. M. Brady, An arabidopsis gene regulatory network for secondary cell wall synthesis, Nature 517 (7536) (2015) 571-5. doi:10.1038/nature14099.

[130] W. Verleyen, S. Ballouz, J. Gillis, Measuring the wisdom of the crowds in network-based gene function inference, Bioinformatics 31 (5) (2015) 745-52. doi:10.1093/bioinformatics/btu715.

[131] C.-H. Yeang, H. C. Mak, S. McCuine, C. Workman, T. Jaakkola, T. Ideker, Validation and refinement of gene-regulatory pathways on a network of physical interactions, Genome Biol 6 (7) (2005) R62. doi: $10.1186 / \mathrm{gb}-2005-6-7-\mathrm{r} 62$

[132] M. Schrynemackers, R. Küffner, P. Geurts, On protocols and measures for the validation of supervised methods for the inference of biological networks, Front Genet 4 (2013) 262. doi:10.3389/fgene.2013.00262

[133] J. Davis, G. M, The relationship between pr and roc curves, in: International Conference on Machine Learning, 2006.

[134] J. Qian, J. Lin, N. M. Luscombe, H. Yu, M. Gerstein, Prediction of regulatory networks: genome-wide identification of transcription factor targets from gene expression data, Bioinformatics 19 (15) (2003) 1917-26.

[135] F. Mordelet, J.-P. Vert, Sirene: supervised inference of regula- 
tory networks, Bioinformatics 24 (16) (2008) i76-82. doi:10.1093/

[136] C. J. Wolfe, I. S. Kohane, A. J. Butte, Systematic survey reveals general applicability of "guilt-by-association" within gene coexpression networks, BMC Bioinformatics 6 (2005) 227. doi:10.1186/1471-2105-6-227.

[137] S. Y. Rhee, M. Mutwil, Towards revealing the functions of all genes in 1175 plants, Trends Plant Sci 19 (4) (2014) 212-21. doi:10.1016/j.tplants . 2013.10 .006 